\title{
Antoine Chalvin : Les Setos d'Estonie
}

Éditions Armeline, 2015

\section{András Kányádi}

\section{(2) OpenEdition}

\section{Journals}

Édition électronique

URL : https://journals.openedition.org/efo/7006

DOI : $10.4000 /$ efo. 7006

ISSN : 2275-1947

Éditeur

INALCO

Édition imprimée

ISBN : 978-2-343-12463-6

ISSN : 0071-2051

Référence électronique

András Kányádi, «Antoine Chalvin : Les Setos d'Estonie », Études finno-ougriennes [En ligne], 48 | 2017 mis en ligne le 17 juillet 2017, consulté le 08 juillet 2021. URL : http://journals.openedition.org/efo/ 7006 ; DOI : https://doi.org/10.4000/efo.7006

Ce document a été généré automatiquement le 8 juillet 2021.

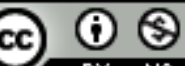

Études finno-ougriennes est mis à disposition selon les termes de la Licence Creative Commons Attribution - Pas d'Utilisation Commerciale 4.0 International. 


\section{Antoine Chalvin : Les Setos d'Estonie}

Éditions Armeline, 2015

\section{András Kányádi}

1 Hansa, en lisant ce mot, on pense inévitablement à la forme latine de la Ligue hanséatique, cette association des villes marchandes de l'Europe du Nord, évincée à peu près autour de la guerre de Trente Ans. Et pourtant, chez Antoine Chalvin, c'est de la gnôle, et pas n'importe laquelle: distillée à partir de farine d'orge, il s'agit d'une trouvaille bien nordique mais datant d'une autre époque belliqueuse, la Grande Guerre, née sous l'impulsion de la pénurie de vodka au Setomaa, c'est-à-dire le pays des Setos. On s'interroge alors sur cette ingénieuse communauté, habitant une contrée à résonance pour le moins enchanteresse.

2 Publié aux éditions Armeline, le livre d'Antoine Chalvin, les Setos d'Estonie, examine sous toutes les coutures ce petit «peuple» septentrional «en péril». On ne peut que (re)saluer l'initiative de l'éditeur breton de faire connaître différentes minorités européennes menacées par la disparition, et se réjouir de l'importance que la maison accorde aux études finno-ougriennes: sur les six livres parus dans cette même collection, quatre leur sont désormais consacrés, et ce grâce aussi (ou surtout) à la «trilogie » de Bernard Le Calloc'h portant sur les communautés hungarophones des Csángós, des Sicules et celle des Sabbataires.

3 Dans la perspective d'un travail optimal de vulgarisation, les auteurs de la série reçoivent carte blanche pour élaborer eux-mêmes la structure de leur ouvrage, en marge d'un triple volet « histoire-langue-culture ». Antoine Chalvin, linguiste aguerri et professeur d'estonien à l'Inalco, a un faible pour la langue, critère souvent décisif dans l'autoreprésentation d'une communauté dont la reconnaissance ne fait pas l'unanimité. Dès lors, il analyse de manière convaincante les différences entre l'estonien et le seto. On observe cette démarche heuristique tout au long du livre.

4 L'ouvrage se compose de onze chapitres dont quatre sont consacrés à l'histoire des Setos, présentée selon une périodisation arrimée à l'histoire de l'Estonie : la période d'avant 1917, la période estonienne de l'entre-deux-guerres, le demi-siècle de l'époque soviétique et, une nouvelle fois, l'ère de l'Estonie indépendante. L'auteur retrace l'évolution de la conscience identitaire et montre les enjeux de l'instrumentalisation 
des Setos opérée tantôt par les Estoniens, tantôt par les Russes. Des cartes contribuent à comprendre la situation particulière de cette petite ethnie à cheval sur deux pays, vivant au sud du lac Pskov et considérée le plus souvent comme formant la branche orthodoxe des Estoniens. Le lecteur a l'occasion de se familiariser avec les diverses théories sur leur ethnogenèse et leur ethnonyme mais peut aussi suivre, grâce aux documents visuels, les différents découpages administratifs de ce territoire qui change assez souvent de maître. De nombreuses données statistiques viennent illustrer la fluctuation démographique, d'autres témoignent de la revitalisation culturelle déclenchée après la chute du totalitarisme.

5 Le chapitre linguistique (chap.5) situe la langue seto parmi les dialectes de l'estonien du Sud, appelés vorro-seto. Selon de nombreux linguistes, il s'agirait néanmoins d'une langue à part entière, avec trois variantes régionales, parlée aujourd'hui par environ 12000 personnes. La reconnaissance officielle du seto reste assez limitée car il est considéré non pas comme une langue régionale mais comme une forme régionale de l'estonien. Pourtant, sur un plan linguistique, la demande de changement de statut semble légitime: le seto possède une voyelle vélaire et des consonnes affriquées absentes de l'estonien standard, il conserve, à l'instar des grandes langues finno-ougriennes, l'harmonie vocalique et présente plusieurs particularités dans la déclinaison et la conjugaison, sans parler du lexique où un bon nombre de mots sont incompréhensibles pour les Estoniens. Un signe distinctif très important concerne la position de la particule de négation, placée après le verbe, contrairement à l'estonien qui la place toujours avant. Faudrait-il y voir, au-delà de l'évolution historique, la marque symbolique d'une protestation identitaire?

6 Le chapitre sur la culture matérielle (chap. 6.) nous réserve aussi quelques surprises ; les Setos vivent beaucoup plus regroupés dans leur village que les Estoniens et possèdent un formidable bijou - des broches d'une taille énorme, en forme de cône incurvé - qui reflète l'âge des femmes, tout en étant la principale marque du statut d'épouse. Quelques photos en couleur, dont celle de la couverture, donnent envie au lecteur de les découvrir in situ. La tradition alimentaire (chap.7) fait en revanche beaucoup moins rêver : flocons d'avoine fermentés pendant deux jours, soupe à l'orge et aux pommes, crêpes à la bière et à la crème aigre ne font venir l'eau à la bouche qu'aux amateurs de défis culinaires. L'idée de présenter une sélection de recettes traditionnelles rassemblées par une parlementaire reste certainement très originale, et le sõir (une sorte de fromage blanc), comme le karask (à mi-chemin entre pain et gâteau) semblent tout à fait abordables.

$7 \quad$ Le code culturel par excellence seto est sans doute le chant polyphonique (leelo), inscrit sur la liste du patrimoine culturel immatériel de l'humanité, qui fait l'objet du chapitre 8 de l'ouvrage. Pratiqué surtout par les femmes, il est aujourd'hui institutionnalisé et mobilise un grand nombre de chorales (plus d'une vingtaine en Estonie et deux en Russie), dont les plus connues enchaînent concerts à l'étranger et enregistrements de disques. Conçu sur l'alternance contrastée d'une voix haute et d'un chœur des voix basses, le leelo tire son nom des refrains onomatopéiques. Si les paroles du chant favorisent l'allitération et le parallélisme, elles peuvent aussi faire allusion au contexte politique, mais dans une grande liberté d'improvisation. En 1986, en l'honneur des meilleures chanteuses du pays Seto, une statue a été édifiée dans le village d'Obinitsa, appelée "la mère du chant», suscitant, à n'en pas douter, plus d'enthousiasme que l'infortuné soldat de bronze de Tallinn. 
8 Le chapitre 9 porte sur le folklore narratif et sur l'épopée. Là encore, l'intérêt de l'auteur est particulièrement vif et particulièrement justifié, puisque Antoine Chalvin est le traducteur français de Kalevipoeg. Les contes setos se distinguent, entre autres, des contes estoniens par la présence d'allitérations, de passages en prose rythmée mais aussi par le nombre élevé de motifs empruntés aux Russes. Il existe même des contes inclassables dans le répertoire pourtant exhaustif d'Aarne-Thompson, dont un, très original, qui détaille les fâcheuses conséquences de la préparation de la bouillie avec du charbon. En revanche Peko, l'épopée paysanne composée de 8000 vers et créée sur commande par la plus célèbre chanteuse illettrée, Anne Vabarna, dans le but d'éveiller la conscience nationale de son peuple, n'a pu être publiée que 70 ans après sa création. De quoi ralentir la renaissance identitaire.

9 L'avant-dernier chapitre de l'ouvrage constitue une esquisse d'anthropologie religieuse. Le culte de Peko, cette divinité païenne de la fertilité et inspiratrice d'épopée, creuse une nouvelle fois l'écart entre Setos et Estoniens. L'auteur nous donne une description circonstanciée de la seule statue de ce dieu (conservée au Musée national estonien à Tartu) ainsi que des rites printaniers et automnaux organisés en son honneur qui ont perduré jusque dans la première moitié du $\mathrm{xx}^{\mathrm{e}}$ siècle. Les fêtes calendaires font preuve de syncrétisme, témoignant d'une synthèse originale entre religion orthodoxe et paganisme ; une longue liste, allant de la "Fête des oiseaux » jusqu'au jour de « Marie du chou », illustre la richesse des diverses pratiques religieuses setos. Des coutumes matrimoniales on retiendra la première nuit des jeunes mariés : dans la grange, et dans un lit sous lequel on plaçait des haches censées favoriser la fertilité. Quant aux coutumes funéraires, mentionnons la plus courante, d'origine préchrétienne, qui a disparu dans les autres régions estoniennes : après l'enterrement, on s'installe pour manger et boire autour de la tombe du défunt. Et l'on y revient, en bon orthodoxe, 40 jours plus tard.

10 Le dernier chapitre s'intéresse à la construction ludique d'une identité nationale. Depuis une vingtaine d'années, les Setos se sont inventé une république indépendante, un royaume seto, qui ne tient que trois jours par an et qui a pour chef, pour des raisons légendaires, un vice-roi. Le sérieux de l'entreprise est honoré parfois par la visite à titre privé du président de la République ou du Premier ministre estonien, qui assistent, émus, à la levée du drapeau et à l'hymne seto. Dans une ambiance d'allégresse, le premier samedi d'août, se déroulent au Setomaa des concours de force, de nourriture et même de hansa; la journée s'achève sur une soirée dansante ou sur un concert, précédés par l'élection du nouveau vice-roi. Le texte de l'hymne et l'analyse de la symbolique du drapeau setos ne sauraient manquer au livre d'Antoine Chalvin qui, dans sa conclusion, souligne avec justesse la fragilité de l'identité seto à notre époque : malgré les signes indéniables de sa revitalisation, elle est de plus en plus exposée à l'assimilation estonienne.

11 Varié, coloré, richement documenté et parfaitement clair dans son propos, les Setos d'Estonie constituent une belle découverte de cette minorité méconnue. Le livre, utile, agréable et ciblant avec succès à la fois le large public et les chercheurs, gagnerait à avoir une meilleure diffusion dans l'espace francophone. Après sa lecture, on ne peut que souhaiter longue vie à la hansa et à ses joyeux inventeurs. 
INDEX

Index géographique : Estonie, Obinitsa, Pskov, Setomaa motscleset setod, vähemus

nomsmotscles Csángós, Estoniens, Russes, Setos, Sicules

Thèmes : popularisation

Keywords : minority, Seto

Mots-clés : minorité, Setos, Hansa, Hanse

Index chronologique : XXe siècle, XXIe siècle (début)

disciplines estonien, seto, võro 